\title{
Generation and characterization of ultra-short electron beams for single spike infrared FEL radiation at SPARC_LAB
}

F. Villa ${ }^{\mathrm{a}, *}$, M.P. Anania ${ }^{\mathrm{a}}$, M. Artioli ${ }^{\mathrm{b}}$, A. Bacci ${ }^{\mathrm{c}}$, M. Bellaveglia ${ }^{\mathrm{a}}$, F.G. Bisesto ${ }^{\mathrm{a}}$, A. Biagioni ${ }^{\mathrm{a}}$, M. Carpanese ${ }^{\text {d }}$, F. Cardelli ${ }^{a}$, G. Castorina ${ }^{a}$, E. Chiadroni ${ }^{a}$, A. Cianchi ${ }^{\mathrm{e}}$, F. Ciocci ${ }^{\mathrm{d}}$, M. Croia ${ }^{\mathrm{a}}$, A. Curcio ${ }^{a}$, G. Dattoli ${ }^{\mathrm{d}}$, A. Gallo ${ }^{\mathrm{a}}$, D. Di Giovenale ${ }^{\mathrm{a}}$, E. Di Palma ${ }^{\mathrm{d}}$, G. Di Pirro ${ }^{\mathrm{a}}$, M. Ferrario ${ }^{\mathrm{a}}$,

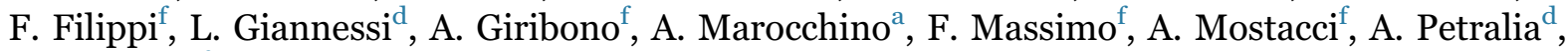
M. Petrarca ${ }^{f}$, V. Petrillo ${ }^{g}$, L. Piersanti ${ }^{a}$, S. Pioli ${ }^{a}$, R. Pompili ${ }^{a}$, S. Romeo ${ }^{a}$, A.R. Rossi ${ }^{c}$, J. Scifo $^{a}$, V. Shpakov ${ }^{a}$, C. Vaccarezza ${ }^{a}$

a INFN-Laboratori Nazionali di Frascati, Via E. Fermi, 40, 00044 Frascati, Italy

b ENEA-Centro Ricerche Bologna, Via Martiri Monte Sole, 4, 40129 Bologna, Italy

c INFN-Milano, Via Celoria, 16, 20133 Milano, Italy

a ENEA-Centro Ricerche Frascati, Via E. Fermi, 45, 00044 Frascati, Italy

e INFN-Roma Tor Vergata and Università di Roma Tor Vergata, Via della Ricerca Scientifica, 1, 00133 Roma, Italy

${ }^{\mathrm{f}}$ INFN-Roma 1 and Università di Roma La Sapienza, Piazzale A. Moro, 2, 00185 Roma, Italy

g INFN-Milano and Università degli Studi di Milano, Via Celoria, 16, 20133 Milano, Italy

\section{A R T I C L E I N F O}

Keywords:

FEL

Single spike

Ultrashort

\begin{abstract}
A B S T R A C T
The technique for producing and measuring few tens of femtosecond electron beams, and the consequent generation of few tens femtoseconds single spike FEL radiation pulses at SPARC_LAB is presented. The undulator has been used in the double role of radiation source and diagnostic tool for the characterization of the electron beam. The connection between the electron bunch length and the radiation bandwidth is analyzed.
\end{abstract}

\section{Introduction}

Ultrashort electron beams, with time duration of tens/few femtoseconds or shorter, enable a wide number of new experiments, that range from the production of single spike high brilliance free-electron laser (FEL) radiation, to the acceleration of high brightness beams in plasma-based acceleration experiments, driven either by electron or laser beams.

A high quality electron beam is mandatory for all applications and the technology that provides the best performances is based on high brightness photo-injectors. FELs driven by ultrashort electron bunches are capable of lasing, up to the x-rays, high brightness pulses [1,2], with enough photons per shot to detect ultrafast processes with pulse duration shorter than the characteristic time of structural damages of the samples. The ultrashort radiation pulses are, indeed, important for the imaging of biomedical tissues and molecules [3], as well as for the time resolved analysis of the atomic surface and plasma dynamics [4]. Various schemes, aimed at enabling ultrashort electron beams to irradiate high brightness FEL radiation pulses, have been recently proposed and tested. Some of these techniques require the spoiling of part of the electron beam, consisting in the selection of a fraction of the bunch for the lasing [5-9], also implemented to produce two-color radiation for pump and probe experiments [10]. The idea of using an undulator tapering to compensate the electron time-energy chirp has been conceived [11,12] and demonstrated in the visible wavelength range $[13,14]$. Short pulses have also been generated in seeded or cascaded FELs [15-19] up to the soft x-ray's range, with improved shot to shot stability and coherence. More sophisticated seeding conceits [20-22] foresee few-cycle seed pulses, but with similar limitation in terms of gain bandwidth. Different proposals, adapted to FELs from mode-locked cavity lasers [23] or exploiting comb-like electron beams [24-26], have been studied for reducing the characteristic time scales of $\mathrm{x}$-ray pulses beyond the FEL bandwidth limitation. Other scheme for ultrashort FEL pulses include electron modulation inside a wiggler magnet by a laser pulse $[27,28]$ or the exploitation of a transversally tilted beam [29].

Another method for the generation of short pulse radiation relies on the compression of electron bunches of very low charge [30]. If the electron beam charge is reduced down to the $\mathrm{pC}$ range, the space charge effects are then so low, that, when the bunch time duration is

\footnotetext{
* Corresponding author.
} 
compressed approaching the fs domain, the electron beam can preserves high transverse and longitudinal quality if properly matched. Such bunches are particularly suitable for Laser WakeField Accelerators (LWFA) and Plasma WakeField Accelerators (PWFA), where dimensions of the electron beam well below the plasma wavelength and low emittances are required. Furthermore, ultrashort electron bunches generate ultrabright FEL pulses. If the electron beam full width is of the order of or shorter than $2 \pi$ times the cooperation length $L_{c}=\lambda_{\text {rad }} / 4 \pi \sqrt{3} \rho\left(\lambda_{\text {rad }}\right.$ being the resonant radiation wavelength and $\rho$ the FEL Pierce parameter [31]), and if it is extracted from the undulator at the beginning of the saturation (before the formation of additional spikes due to superradiance [32]) the FEL emission occurs in the single spike regime and the pulses are nearly Fourier transformlimited, with coherent phases. This technique, even if exploits a straightforward concept, is highly demanding for the electron bunch diagnostics at such low charges and length. The milestone experiment at low charge was performed at the Linac Coherent Light Source (LCLS), where the operation mode at $20 \mathrm{pC}$ has been explored [33], and FEL $\mathrm{x}$-rays emitted with typical duration of a few femtoseconds $[6,34]$, opening perspectives also at other facilities $[35,36]$.

As preparatory phase of the LWFA/PWFA experiments to be performed at SPARC_LAB [37,38], electron beams of charge as low as $20 \mathrm{pC}$ and RMS bunch duration down to $30 \mathrm{fs}$ have been produced and measured. After the transport in the undulator, single spike infrared pulses were observed and detected in the single-pass FEL [39]. Accurate spectral acquisitions of the radiation were crosschecked with the direct measurements of the length of the electron beam, in order to control their mutual consistence. In this proceeding, the technique for producing and measuring few tens fs long electron beams, and the consequent generation of single spike FEL radiation at SPARC_LAB are reported. The undulator has been used in the double role of radiation source and supplementary diagnostics for the characterization of the electron beam. The relationship between the electron bunch length and the radiation bandwidth is therefore discussed.

\section{Electron bunch generation and characterization}

We report here on the single spike FEL radiation as obtained in two experimental shifts, referred to as beam (1) and (2), with similar RF compression parameter, done at the SPARC_LAB test facility (Fig. 1) [38], separated by about one month.

SPARC_LAB photoinjector is composed by an S-band RF gun followed by three S-band travelling wave (TW) cavities that can accelerate the beam up to $180 \mathrm{MeV}$ on crest or $120 \mathrm{MeV}$ in velocity

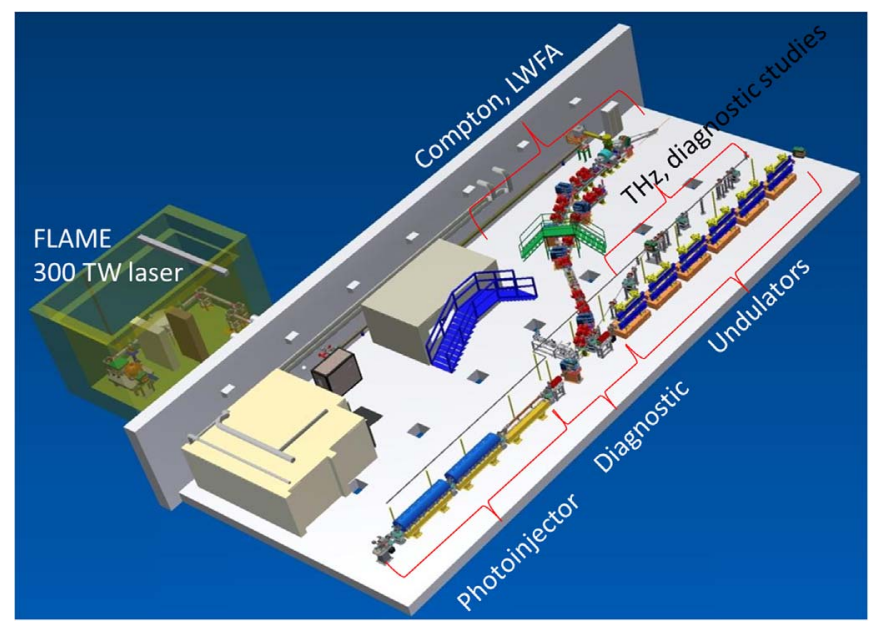

Fig. 1. SPARC LAB facility layout. The experiment was done in the straight line from the photoinjector to the undulators.
Table 1

Electron beam parameters for beam (1) and (2).

\begin{tabular}{lll}
\hline Electron beam parameters & Beam (1) & Beam (2) \\
\hline Charge (pC) & $20 \pm 1$ & $22 \pm 1$ \\
Mean energy (MeV) & $113.93 \pm 0.03$ & $111.80 \pm 0.03$ \\
Energy spread (KeV) & $115 \pm 15$ & $73 \pm 19$ \\
Bunch RMS length (fs) & $33 \pm 5$ & $38 \pm 5$ \\
Emittance x (mm mrad) & $1.18 \pm 0.10$ & $0.83 \pm 0.08$ \\
Emittance y (mm mrad) & $1.14 \pm 0.10$ & $0.76 \pm 0.22$ \\
\hline
\end{tabular}

bunching compression [40]. The experiment started with the photogeneration of a sub-ps electron beam, obtained by illuminating the cathode with a laser pulse at $264 \mathrm{~nm}, 300 \mathrm{fs}$ FWHM long, with a flat top transverse profile of $0.5 \mathrm{~mm}$ diameter. We measured $4.93 \pm$ $0.03 \mathrm{MeV}$ of beam energy at the end of the gun. A value of about $20 \mathrm{pC}$ of charge was extracted from the cathode and measured with a beam current monitor. The electron bunch was then propagated along the linac and compressed by means of the velocity bunching technique [40], that chirps and compresses the beam at the same time. The minimum length condition is obtained accurately choosing the accelerating phase of the first TW section. The two experimental shifts were done near the maximum compression, to have very short pulses, with a slightly different compressing factor.

In the diagnostic section at the end of the linac we measured the electron beam parameters, reported in Table 1 . We measured the longitudinal phase space of the beams using a dipole and the vertical deflecting modes of an S-band RF deflecting (RFD) cavity following the procedure described in [41]. With $2 \mathrm{MW}$ power available for our RFD, a camera pixel size equal to $10 \mu \mathrm{m}$ and an imaging magnification of 1:3, the intrinsic limit of resolution is about $20 \mathrm{fs}$ at the working beam energy [41]. The two working points were near the maximum compression and the RMS duration for the electron beam $\sigma_{z}$ was 33 $\pm 5 \mathrm{fs}$ ( $38 \pm 5$ fs for beam (2)), corresponding to a peak current of about $260 \pm 40$ A (245 \pm 30 A for beam (2)).

The measured bunch mean energy was $113.93 \pm 0.01 \mathrm{MeV}$ for beam (1) and $111.69 \pm 0.03 \mathrm{MeV}$ for beam (2). The RMS projected energy spread was respectively $115 \pm 15 \mathrm{KeV}$ and $73 \pm 19 \mathrm{KeV}$. Due to the extremely low value of the charge of the electron beam, the second order correlations in the phase space were negligible, so the compression factor can be higher and the energy spread considerably smaller in comparison with hundreds of $\mathrm{pC}$ bunches.

We measured the beam projected emittances $\varepsilon_{x}=1.18 \pm 0.1 \mu \mathrm{m}$ and $\varepsilon_{y}=1.14 \pm 0.1 \mu \mathrm{m}$ for beam (1) $\quad\left(\varepsilon_{x}=0.83 \pm 0.08 \mu \mathrm{m}\right.$ and $\varepsilon_{y}=0.76 \pm 0.22 \mu \mathrm{m}$ for beam (2)) [42] via a quadrupole scan of the electron beams [43].

The parameters of the electron beam in the two cases (1) and (2) are summarized in Table 1, while the phase space of case (1) is presented in Fig. 2.

\section{FEL setup and measurements}

The compressed beam was then propagated in the undulator, which is composed by five variable gap modules of 75 periods each (77 including termination periods), with a period length of $2.8 \mathrm{~cm}$ and a maximum magnetic strength of $K=2.2$, plus one module with $1.4 \mathrm{~cm}$ period length. The first five module gaps of the undulator were tuned at $740 \mathrm{~nm}(747 \mathrm{~nm}$ in case (2)), with $\mathrm{K} \sim 1.8$. While we use the last module for harmonic cascade, in this experiment it was not activated as we focalized our attention on the fundamental harmonic. In the transverse plane the beam was matched to the undulator with Twiss parameters (averaged over the undulator length) $\left\langle\beta_{x}\right\rangle=\left\langle\beta_{y}\right\rangle=1.5 \mathrm{~m}$ and measured projected emittances.

With the electron parameter listed in Table 1, the 1D FEL parameter is evaluated to be $\rho_{1 D}=7.8^{*} 10^{-3}$ for beam (1) $\left(\rho_{1 D}=8.7^{*} 10^{-3}\right.$ for beam (2)), while the three-dimensional corrections [31], due mainly to diffrac- 

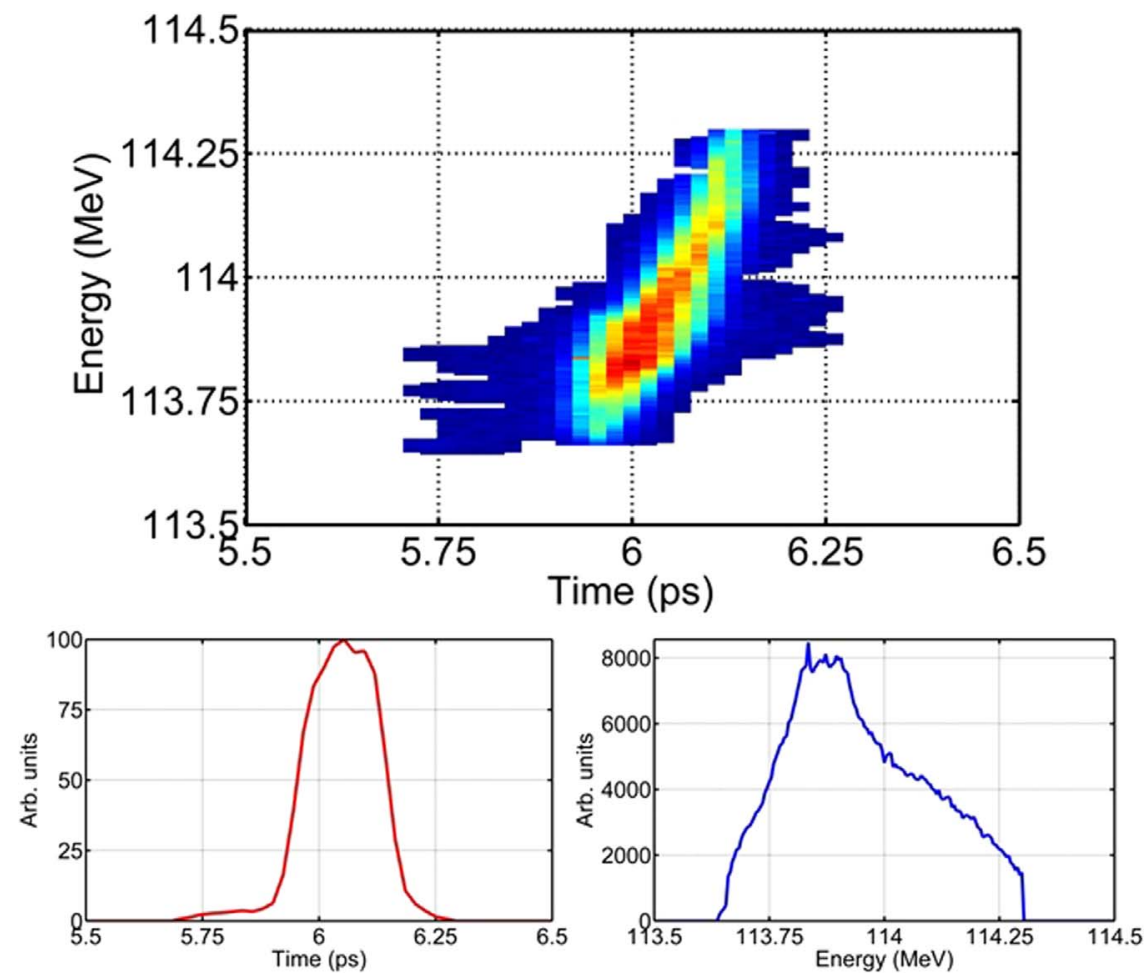

Fig. 2. Measured longitudinal phase space of the electron for beam (1) (with profiles).

tion, decrease it to $\rho_{3 D}=5.1 * 10^{-3}\left(\rho_{3 D}=5.5^{*} 10^{-3}\right.$ for beam (2)). Assuming this last value, the cooperation length is $L_{c}=6.7 \mu \mathrm{m}$ $\left(L_{c}=6.3 \mu \mathrm{m}\right.$ for beam (2)) With those parameters electron beams whose full width $\left(2.35 \sigma_{z}\right)$ is shorter than $2 \pi L_{c}\left(140 f s^{*} c\right.$ for beam (1) and $130 f s^{*} c$ for beam (2)) will drive a clean single spike FEL pulse. This regime, called superradiance [44-46], is characterized by a strong influence of the slippage of the radiation on the electron beam. After the end of the exponential phase, the emission continues with the formation of secondary peaks. The energy grows along the undulator coordinate $z$ with a rate proportional to $z^{2}$ and the spectrum broadens and deteriorates.

The main diagnostics used to measure the output radiation were an in-vacuum spectrometer, called LUXOR and based on an extension of [47], operating in the spectral range $35-800 \mathrm{~nm}$ and a commercial fiber spectrometer with $200-850 \mathrm{~nm}$ spectral range. Sequences of 300 spectra have been collected with each set-up. The data show sharp single spike spectra in nearly $100 \%$ of cases, confirming that $\sigma_{z}<2 \pi L_{c} / 2.35$. The clean shape of the spectral lines demonstrates also that the FEL emission pulses have been acquired at the end of the exponential amplification stage, where the spectrum is characterized by a multipeaked shape, and before the onset of additional spikes due to superradiance effects [32]. The comparison between LUXOR with fiber spectrometers average data for beam (1) is shown in Fig. 3. The two spectrometers give very similar distributions and mean values. The main parameters of the radiation spectra have been reported in Table 2. We can assume as typical value of the RMS bandwidth in case (1) the average between the values of the two spectrometers, $\sigma_{\lambda}=4.75 \mathrm{~nm}$. In case (2) only the fiber spectrometer was used and $\sigma_{\lambda}=4.6 \mathrm{~nm}$.

We have performed GENESIS 1.3 [48] simulations fixing all the electron parameters to the measured values (as in Table 1) but changing the longitudinal RMS lengths between 20 and $50 \mathrm{fs}$. We assumed constant parameters along the longitudinal direction in the simulation, finding a good agreement with experimental data. We performed 100 runs for each point changing the seed for the shot noise to obtain information on the statistical variation of the parameters. A sample of the simulated radiation spectrum is shown in Fig. 2, with good agreement with the experimental data, while the average spec-

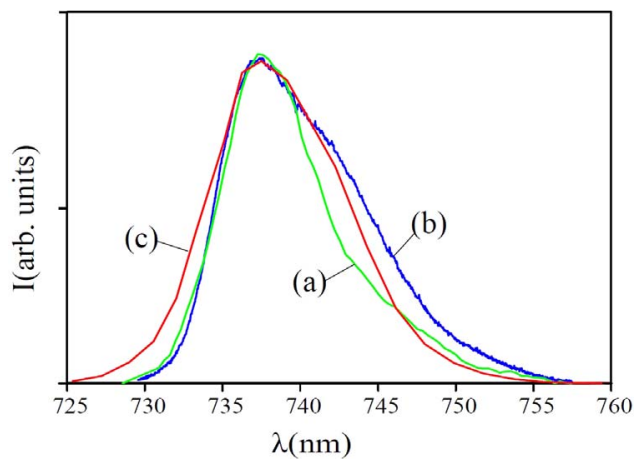

Fig. 3. Averaged FEL spectra for LUXOR spectrometer (a), fiber spectrometer (b) and simulation with GENESIS (c) for the beam (1).

Table 2

FEL spectral measurements for beam (1) and (2). Experimental errors take in account statistical variations and the simulation errors represent statistical variation over random seeds for the shot noise.

\begin{tabular}{lll}
\hline Spectrometer & $<\lambda>(\mathrm{nm})$ & $\left.<\sigma_{\lambda}\right\rangle(\mathrm{nm})$ \\
\hline LUXOR (1) & $739.9 \pm 1.3$ & $4.74 \pm 0.27$ \\
Fiber (1) & $739.8 \pm 1.7$ & $4.76 \pm 0.36$ \\
Simulation (1) & $740 \pm 0.9$ & $4.75 \pm 0.06$ \\
Fiber (2) & $747 \pm 1$ & $4.6 \pm 0.5$ \\
Simulation (2) & $748 \pm 0.9$ & $4.65 \pm 0.06$ \\
\hline
\end{tabular}

trum and bandwidth are reported in Table 2 .

In Fig. 4 we have reported the measured electron bunch lengths and the measured bandwidths (for beam (1) both bandwidths are reported). The black dots in Fig. 4 are the output of the simulations evaluated at the onset of saturation. The simulation results are in good agreement with the experimental data. The white points in Fig. 4 represent the scaling law of the bandwidth at saturation $\sigma_{\lambda} \propto \sqrt{\rho / N}(\mathrm{~N}$ being the number of undulator period at saturation) [49]. The system reaches saturation in about $L_{\text {sat }} \approx 10 L_{\text {gain }}$, corresponding to a number of 


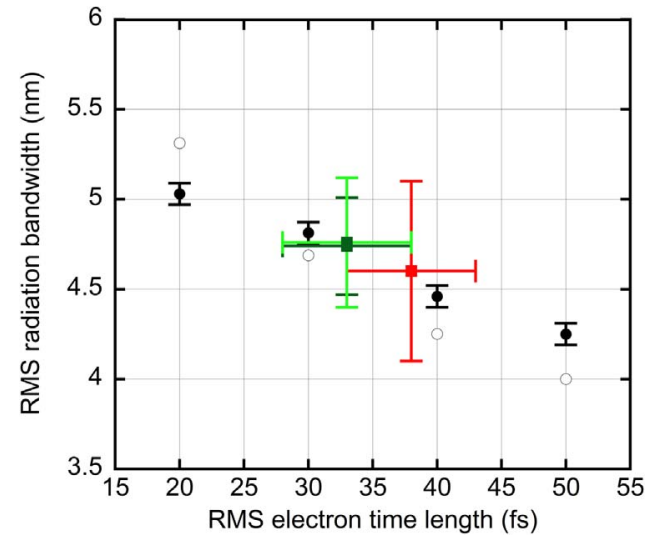

Fig. 4. The plot shows the RMS bandwidth of the FEL radiation versus electron RMS bunch length. The white points are the best fit with the scaling law $\sigma_{\lambda} \propto \sigma_{z}^{-1 / 3}$, the black points are GENESIS simulations and the squares are the two experimental points, green for beam (1) and red for beam (2). For beam (1), the bars on the bandwidth represent the measurements with LUXOR (darker) and fiber (lighter) spectrometers. (For interpretation of the references to color in this figure legend, the reader is referred to the web version of this article.)

periods $N \propto 1 / \rho$ [49]. The bandwidth $\sigma_{\lambda}$ is therefore proportional to $\rho$ and, as a function of the length of the electron beam, $\sigma_{\lambda} \propto \sigma_{z}^{-1 / 3}$. If the electron beam is much shorter than $L_{c}$, the saturation is anticipated due to the slippage by a factor scaling with $\sigma_{z} / L_{c}$ and the bandwidth scaling law turns out to depend on the electron length as $\sigma_{\lambda} \propto \sigma_{z}^{-2 / 3}$ [46]. The radiation is also emitted in a near Fourier transform-limited condition, as the limit for a Gaussian pulse is $\sigma_{z} \sigma_{k}=2.35^{2} \sigma_{z} \sigma_{\lambda} / \lambda^{2}=0.44$ [50] and we obtain $\sigma_{z}=31 \pm 2 f s^{*} c\left(\sigma_{z}=32 \pm 4 f s^{*} c\right.$ for beam (2)), in good agreement with our measurements.

While the radiation is single spiked, it is not statistically coherent because it grows from the initial electron noise. The RMS intensity fluctuations reach about $100 \%$ of the average peak intensity, although the fluctuations in average wavelength and bandwidth are respectively $0.2 \%$ and $6.6 \%(0.12 \%$ and $11 \%$ for beam (2)).

This method is very good to extract the time duration of ultrashort electron bunches, as the FEL radiation bandwidth produced by such pulses is large enough to have very good resolution over the spectral measurement. This method is capable of measuring bunch shorter than the resolution of our RFD and it is easily scalable to shorter wavelengths. The data analysis is very performing where the analytical solution is properly working (i. e. for pulses shorter than $L_{c}$ and near saturation, where the bandwidth has a simple formulation), but when the pulse exhibits a multipeaked spectrum, due to superradiance or in exponential gain regime, or when the electron parameters change significantly over the longitudinal dimension a more complex data analysis with simulations (i. e. GENESIS) should be done.

In conclusions, we have produced ultrashort electron bunches and measured their length. The single spike FEL emission has been studied, giving insight in the superradiant regime. Spectral measurements permit to retrieve the value of the electron bunch length with a good agreement. From the diagnostic point of view, we tested the possibility to measure low charged ultrashort electron bunches and we propose a method to extend the duration evaluation of high brightness ultrashort electron beams even to shorter bunches using the FEL bandwidth.

\section{Acknowledgments}

This work has been partially supported by the EU Commission in the Seventh Framework Program, Grant Agreement 312453 EuCARD2and by the Italian Ministry of Research in the framework of FIRB Fondo per gli Investimenti della Ricerca di Base, Project no. RBFR12NK5K

\section{References}

[1] P. Emma, et al., Nat. Phot. 4 (2010) 641

[2] T. Ischikawa, et al., Nat. Phot. 6 (2012) 540.

[3] A. Aquila, et al., Struct. Dyn. 2 (2015) 041701.

[4] F. Krausz, M. Ivanov, Rev. Mod. Phys. 81 (2009) 163.

[5] P. Emma, et al., Phys. Rev. Lett. 92 (2004) 074801.

[6] Y. Ding, et al., Phys. Rev. Lett. 109 (2012) 254802.

[7] T. Tanaka, Phys. Rev. Lett. 110 (2013) 084801.

[8] E. Prat, S. Reiche, Phys. Rev. Lett. 114 (2015) 244801.

[9] A. Marinelli, et al., Phys. Rev. Lett. 116 (2016) 254801.

[10] A.A. Lutman, et al., Phys. Rev. Lett. 110 (2013) 134801.

[11] E.L. Saldin, et al., Phys. Rev. ST Acc. Beams 9 (2006) 050702.

[12] T. Tanaka, Phys. Rev. Lett. 114 (2015) 044801.

[13] L. Giannessi, et al., Phys. Rev. Lett. 106 (2011) 144801.

[14] G. Marcus, et al., Appl. Phys. Lett. 101 (2012) 134102.

[15] T. Watanabe, et al., Phys. Rev. Lett. 98 (2007) 034802.

[16] L. Giannessi, et al., Phys. Rev. Lett. 108 (2012) 164801.

[17] M. Labat, et al., Phys. Rev. Lett. 107 (2011) 224801.

[18] L. Giannessi, et al., Phys. Rev. Lett. 110 (2013) 044801.

[19] E. Allaria, et al., Nat. Phot. 6 (2012) 699.

[20] A.A.Zholents and W.M. Fawley, Phys. Rev. Lett. 92 (2004) 224801.

[21] E.L. Saldin, et al., Opt. Comm. 239 (2004) 161.

[22] D. Xiang, et al., Phys. Rev. ST Accel. Beams 12 (2009) 060701.

[23] D.J. Dunning, et al., Phys. Rev. Lett. 110 (2013) 104801.

[24] V. Petrillo, et al., Phys. Rev. Lett. 111 (2013) 114802.

[25] A. Marinelli, et al., Nat. Comm. 6 (2015) 6369.

[26] A. Petralia, et al., Phys. Rev. Lett. 115 (2015) 014801.

[27] A.A. Zholents, Phys. Rev. ST Accel. Beams 8 (2005) 040701.

[28] A.A.Zholents and G. Penn, Phys. Rev. ST Accel. Beams 8 (2005) 050704.

[29] E. Prat, et al., Phys. Rev. ST Accel. Beams 18 (2015) 100701.

[30] J.B. Rosenzweig, et al., Nucl. Instr. Meth. Phys. Res. A 593 (2008) 39.

[31] M. Xie, Design optimization for an X-ray free electron laser driven by SLAC linac, presented at 1995 Particle Accelerator Conference, IEEE, Dallas (TX), 1995, p. 183.

[32] R. Bonifacio, et al., Phys. Rev. A 40 (1989) 4467.

[33] Y. Ding, et al., Phys. Rev. Lett. 102 (2009) 254801.

[34] C. Behrens, et al., Nat. Comm. 5 (2014) 3762.

[35] S. Reiche et al., Low charge operation of SwissFEL, presented at FEL $\{C\}$ 2009-31\{C\}st International Free Electron Laser Conference, 2009.

[36] B. Marchetti, et al., Phys. Procedia 52 (2014) 80.

[37] A.R. Rossi, et al., Nucl. Instr. Meth. Phys. Res. A 740 (2014) 60.

[38] M. Ferrario, et al., Nucl. Instr. Meth. Phys. Res. B 309 (2013) 183

[39] L. Giannessi, et al., Phys. Rev. ST Accel. Beams 14 (2011) 060712.

[40] M. Ferrario, et al., Phys. Rev. Lett. 104 (2010) 054801.

[41] A. Cianchi, et al., Phys. Rev. ST Accel. Beams 18 (2015) 082804.

[42] M. Quattromini, et al., Phys. Rev. ST Acc. Beams 15 (2012) 080704.

[43] M.G. Minty, F. Zimmermann, Measurement and Control of Charged Particle Beams, Springer-Verlag, Berlin, 2003.

[44] R. Bonifacio, F. Casagrande, Nucl. Instr. Meth. Phys. Res. A 239 (1985) 36.

[45] R. Bonifacio, et al., Opt. Comm. 68 (1988) 369.

[46] R. Bonifacio, et al., Nucl. Instr. Meth. Phys. Res. A 296 (1990) 358.

[47] L. Poletto, et al., J. Phys. Conf. Ser. 425 (2013) 122011.

[48] S. Reiche, Nucl. Instr. Meth. Phys. Res. A 429 (1999) 243.

[49] R. Bonifacio, et al., Phys. Rev. Lett. 73 (1994) 70.

[50] A.M. Weiner, Ultrafast Optics, John Wiley \& Sons, Inc., Hoboken, New Jersey, 2009. 